\title{
CANNY-BASED METHOD OF IMAGE CONTOUR SEGMENTATION
}

\author{
Diana Zahorodnia, Yuriy Pigovsky, Pavlo Bykovyy \\ Ternopil National Economic University, 3 Peremohy Square, Ternopil, Ukraine, 46020, \\ dza@tneu.edu.ua,pb@tneu.edu.ua
}

\begin{abstract}
The paper presents modification of the image contour segmentation method based on Canny approach. A distinctive feature of the proposed method is the usage of wavelet-functions for contour sharpening during image transformation and the application of wavelet overlay at the beginning. The linear values of the intensity of the image pixels that are covered by wavelet are calculated and the result of wavelet transform is recorded into the central element of the pixels combination. As a result, two matrices of the same size as input image are received.

After that a threshold for pixel intensity values is specified empirically: if the intensity values are less than a threshold, they are changed into 0 , otherwise, they are unchangeable.

The next step is calculation of the gradient according to the Canny method, it is performed for each of two matrices, obtained at the previous stage. As a result, image pixels where a local maximum gradient is obtained could refer to contours. The received contours, based on two matrices, are combined using logical "OR" operation. A set of contours with different levels of hierarchy can be obtained while adjusting the length of the wavelet.

The modified method allowed to obtain a sequence of hierarchical object contours with an adjusted detailing. This method is a part of the object recognition information technology that significantly reduces the size of information processing of video surveillance systems. Copyright (C) Research Institute for Intelligent Computer Systems, 2016. All rights reserved.
\end{abstract}

Keywords: Image contour segmentation; Canny method; wavelet-functions; video surveillance system.

\section{INTRODUCTION}

Advances in information technology made possible increasing of the video surveillance systems functionality without great financial costs. Surveillance systems have been previously implemented mostly in public infrastructure elements such as airports, subways, etc. to monitor the flows of people and transport [1]. However, nowadays such systems are commonly used in the home [2-4].

It is obvious that this approach allows to increase the amount of video sensors as well as video transmitting devices, hardware and software for video processing $[5,6]$. Therefore, methods allowing to reduce the amount of processed information are relevant today.

One of the main goals of the use of modern video processing systems is the interpretation of the image content. In order to do this, it is necessary to separate objects from the background. Segmentation allows to split an image into its constituent parts or objects and separate the object from the background. Thus, you can easily process an image and identify its contents. High quality of image segmentation can be achieved by contour detection. This reduces greatly the amount of data for image processing, and at the same time stores the important information about the objects in the image, such as their shape, size, number [7-13].

Typically, image segmentation algorithms are based on one of the two main properties of the signal brightness: uniformity and brightness breakups [14]. Segmentation methods based on the object contour detection are also often applied $[15,16]$. They refer to segmentation algorithms based on brightness breakups.

A large amount of contour and boundary detection algorithms has been offered and described in scientific literature. The most popular methods include: Roberts, Sobel, Previtta, Kirsch, Robinson operators, Canny algorithm and LoG algorithm [17].

However, nowadays there isn't a universal method or algorithm of image contour detection [18]. To determine the appropriate algorithm for contour detection, the contour orientation and structure should be taken into account as well as the availability and type of noise in the image. Each class of algorithms solves its specific problems and detects efficiently only a certain type of counters. Thus, the issue of the development of the contour image segmentation methods is relevant today. 


\section{THE PROPOSED APPROACH}

The Canny contour detection method [19] was chosen as the basic one among a range of such methods. Its advantages include immunity, and the thickness of the contour line is of 1 pixel. However, this method cannot be applied to a hierarchical approach. Therefore, the implementation of this approach requires morphological analysis of a set of contours that significantly reduces efficiency. In this paper the following hierarchical contour segmentation method was developed.

Image processing by the mask overlay is the most common way of the image contour detection. However, instead of the use of Sobel mask with the Canny method, in this paper wavelets are proposed to use [20-23].

The first step - wavelet overlay.

For a wavelet of $n$ size the procedure is based on calculating of the linear combination of pixel intensity values of the image covered by wavelets.

To improve the convergence of the wavelet transform the utmost row of pixels is complemented by copying pixels onto the wavelet length:

$$
z_{i, j}=\left\{\begin{array}{l}
I_{i, 1}, \text { if } j<n, \\
I_{i, M}, \text { if } j>M+n \\
I_{i, j-n+1}, \text { otherwise }
\end{array}\right.
$$

where $I_{i, j}$ - the original image pixel, $i=1,2, \ldots N$, $j=1,2, \ldots M ; z_{i, j}-$ a pixel of the complemented image, $i=1,2, \ldots N, j=1,2, \ldots M+2 n ; n$ - wavelet length.

For each image pixel a value of the overlaid wavelet onto a row of pixels is determined:

$$
W X_{i, j}=\sum_{k=i-n}^{i-1} w_{k} z_{k, j}+\sum_{k=i+1}^{i+n} w_{k} z_{k, j},
$$

where $z_{k, j}$ - value of pixel brightness (intensity) corresponding to the $w_{k}$ wavelet coefficient. The result of wavelet transform is written onto the central element.

In the same way the image on the OY axis is complemented:

$$
z_{i, j}=\left\{\begin{array}{l}
I_{1, j}, \text { if } i<n, \\
I_{N, j}, \text { if } i>N+n \\
I_{i-n+1, j}, \text { otherwise }
\end{array},\right.
$$

where $I_{i, j}$ - the original image pixel, $i=1,2, \ldots N$, $j=1,2, \ldots M ; z_{i, j}-$ a pixel of the complemented image, $i=1,2, \ldots N+2 n, j=1,2, \ldots M ; n$ - wavelet length.

For each image pixel a value of the overlaid wavelet onto a column of pixels on the OY axis is determined.

$$
W Y_{i, j}=\sum_{k=j-n}^{j-1} w_{k} z_{i, k}+\sum_{k=j+1}^{j+n} w_{k} z_{i, k}
$$

As a result two matrices of the same size as the input image are obtained: $\left[W X_{i, j}\right\rfloor$ and $\left\lfloor W Y_{i, j}\right\rfloor$.

While overlaying wavelet on the OX axis image, the greatest value will be on one pixel thick vertical lines. Moreover, the value on the image areas with constant brightness will be equal to zero as the sum of the wavelet coefficients is equal to zero. Similarly, while overlaying wavelet on the OY axis horizontal lines will be detected most.

The second step - setting a threshold (threshold filter).

A threshold for pixel intensity values is set. If the intensity value is less than a threshold, it is changed into 0 , and the value is not changed in case it is higher than a threshold.

As a result, once again, we obtain two matrices of the same size as the input image: $\left\lfloor W X_{i, j}^{\prime}\right\rfloor$ and $\left\lfloor W Y_{i, j}^{\prime}\right\rfloor$ :

$$
\begin{aligned}
W X_{i, j}^{\prime} & =\left\{\begin{array}{l}
W X_{i, j}, \text { if } W X_{i, j}>P \\
0, \text { otherwise }
\end{array},\right. \\
W Y_{i, j}^{\prime} & =\left\{\begin{array}{l}
W Y_{i, j}, \text { if } W Y_{i, j}>P \\
0, \text { otherwise }
\end{array},\right.
\end{aligned}
$$

where $\mathrm{P}-\mathrm{a}$ threshold value.

Then variations in intensity are found. Variations refer to a set of pixels adjacent to two areas and connected according to a certain criterion.

The third step - calculating morphological gradient.

The gradient value is calculated to determine the jump intensity and its direction. The contour is detected where the image gradient has the maximum value.

The value of the gradient characterizes the rate of the function $f$ change at the point $(\mathrm{x}, \mathrm{y})$ and is calculated by formula: 


$$
\left[\begin{array}{l}
G_{x} \\
G_{y}
\end{array}\right]=\left[\begin{array}{l}
\frac{\partial f}{\partial x} \\
\frac{\partial f}{\partial y}
\end{array}\right],
$$

The direction of the gradient vector coincides with the direction of the maximum rate of the function change at the point $(x, y)$ and is calculated by formula:

$$
\nabla f=\sqrt{G_{x}^{2}+G_{y}^{2}}
$$

This value is equal to the maximum rate of the function $f$ change at the point $(x, y)$. Moreover, the maximum value can be obtained in the direction of the vector $\nabla f$. The value $\nabla f$ is often called gradient. The direction of the gradient vector is also an important characteristic. Let $\alpha(x, y)$ is the angle between the vector direction $\nabla f$ at the point $(\mathrm{x}, \mathrm{y})$ and the axis OX. Therefore,

$$
\alpha(x, y)=\operatorname{arctg}\left(\frac{G_{y}}{G_{x}}\right),
$$

Then, it is easy to find the direction of the contour at the point $(\mathrm{x}, \mathrm{y})$. This contour is perpendicular to the direction of the gradient vector at this point.

Only gradient value local maximum is designated as border. Contour pixels can be defined as those ones, which allow to obtain gradient local maximum in the direction of the gradient vector.

The contours are detected by the suppression of all other contours, which are not connected with certain (strong) contours. To obtain an intermediate value at the previous stage it is necessary to select groups of pixels and refer them to the contour in case they are connected with a certain fixed boundary or, otherwise, suppress them. A pixel is added to the group if it is adjacent to this group in one of the 8 directions.

The following procedure is performed separately for two matrices: $\left[W X_{i, j}^{\prime}\right\rfloor$ and $\left\lfloor W Y_{i, j}^{\prime}\right\rfloor$. The resulting set of contours is received according to the scheme of logical connective "or".

After the receiving of the first contour (top-level hierarchy), the length of a wavelet is reduced to obtain the contour of the next level. Then the procedure of the first step is performed. However, it only involves processing of a part of the image which is in the centre of the contour of a higher hierarchical level.

When choosing the length of the wavelet, geometric dimensions of the objects that compose the image should be taken into account. In the first phase the wavelet length must meet the geometric dimensions of the "largest object" and it should be reduced at the next level to fit the object. The desired level of specification can be considered as a criterion of the exit from the cycle. The degree of specification depends on the purpose of the given problem, that is, segmentation should be discontinued when the objects of interest are selected.

Among the advantages of the proposed method of object selection are closed contours and wavelet overlay that allow to ignore all the contours of the image details which are smaller than the length of the wavelet and thus, the image can be divided into a set of hierarchical contours ("contour - subcontour").

\section{PRACTICAL IMPLEMENTATION}

The proposed method has been implemented in Matlab software package as m-executable scripts.

Increasing conversion is performed using the Haar wavelet (Fig. 1) as it has a low computational complexity and does multiplication by +1 and -1 , that greatly simplifies its hardware implementation and therefore increases efficiency.
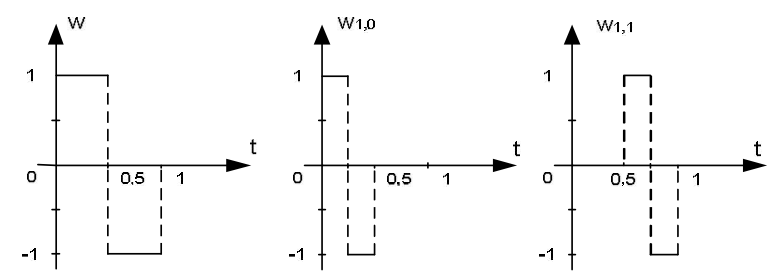

Fig. 1 - Haar wavelets

$w(t)$ function is called Haar function (Fig. 1). The above-mentioned functions can be obtained through transformation (compression and tension along the horizontal axis of abscissas) and shifting of the argument function $w(t)$ which is determined by formula:

$$
w(t)=\left\{\begin{array}{l}
1, t \in[0,1 / 2), \\
-1, t \in[1 / 2,1), \\
0, t \notin[0,1) .
\end{array}\right.
$$

An example of the test image is shown in Fig. 2. While creating this image the peculiarities of the subject area were taken into account. The image reproduces a set of the characteristic features of real objects in black, white and gray areas and it can be representative, that is, an average quality of real objects processing can be determined according to the degree of its efficiency and accuracy. 


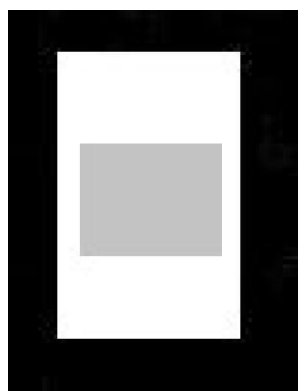

Fig. 2 - Test image
Fig. 3 shows an image band, a graph of its pixels intensity $\left(I_{61, j}\right)$; the same image band with the overlaid Haar wavelet, a graph of its pixels intensity $\left(W X_{61, j}\right)$ and a graph which is formed after the setting of a threshold $W X_{61, j}^{\prime}$.

Fig. 4 shows the image obtained after the wavelet overlay onto the all image pixels: a) -along the rows, c) -along the columns; image with thresholding $(b, d)$.

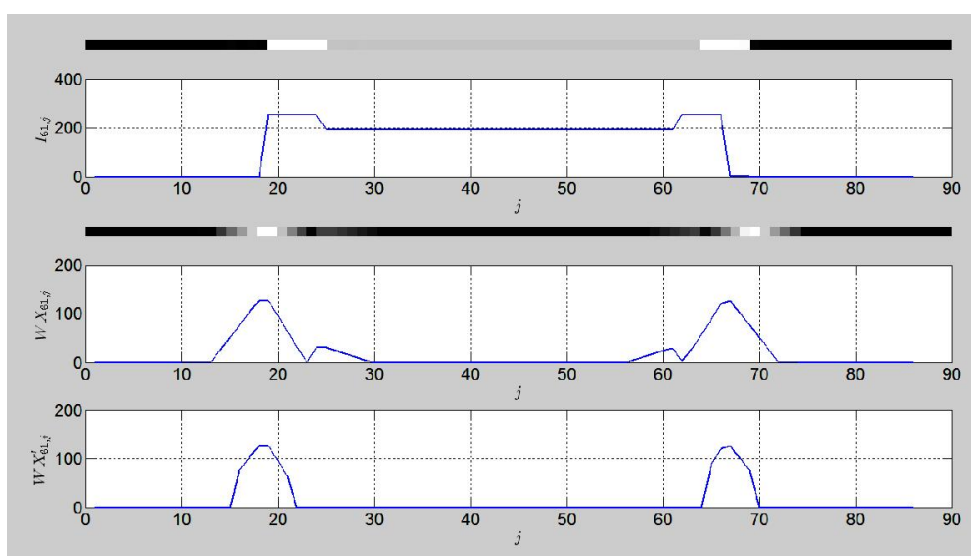

Fig. 3 - An image band and intensity graphs

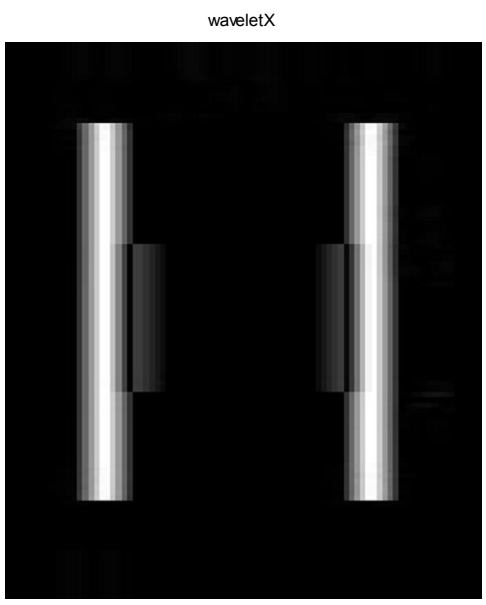

a)

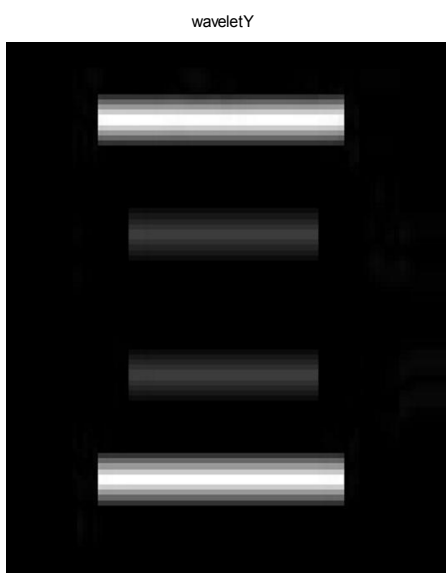

c)

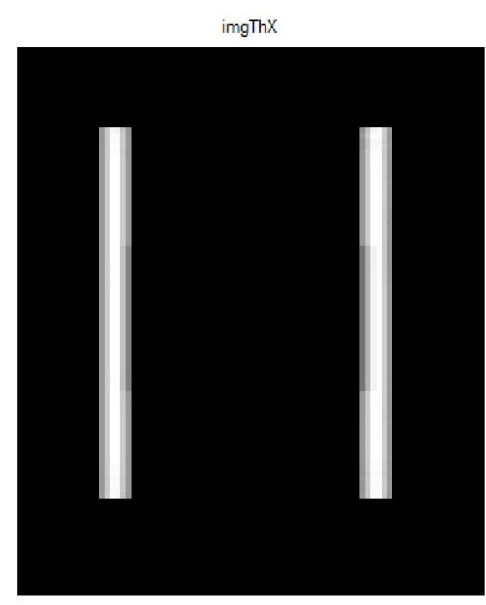

b)

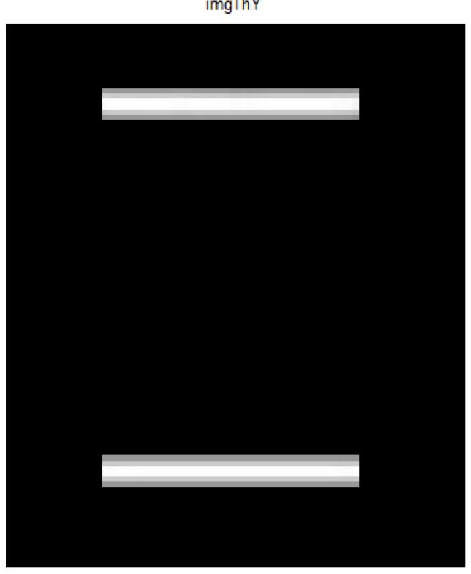

d)

Fig. 4 - Images obtained after the wavelets overlay: a) -along the rows, c) - along the columns; b), d) - image with thresholding 
As a result, an image is obtained at the first hierarchical level which is shown in Fig. 5; the next level of the hierarchy is shown in Fig. 6. The contours are detected due to the classic Canny method in Fig. 7.

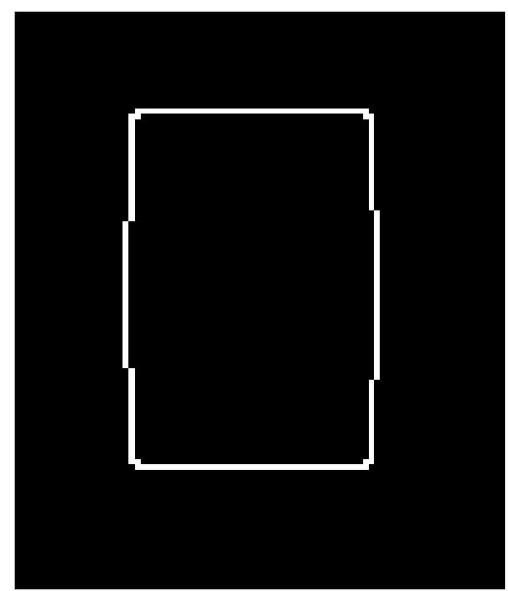

Fig. 5-Contours detection at the first level of the hierarchy

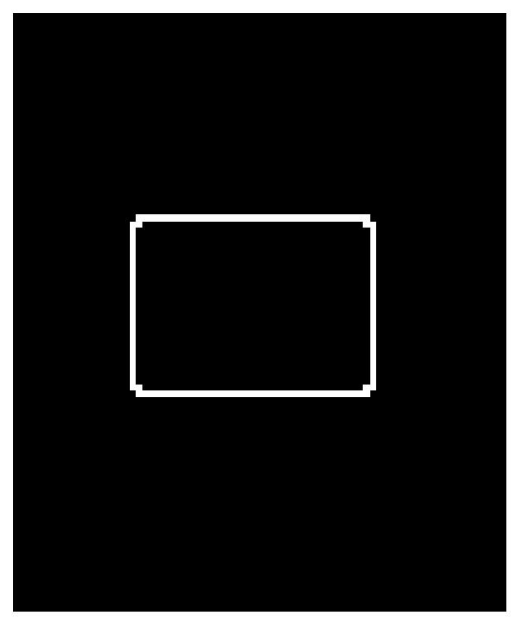

Fig. 6 -Image contour obtained at the second level of the hierarchy

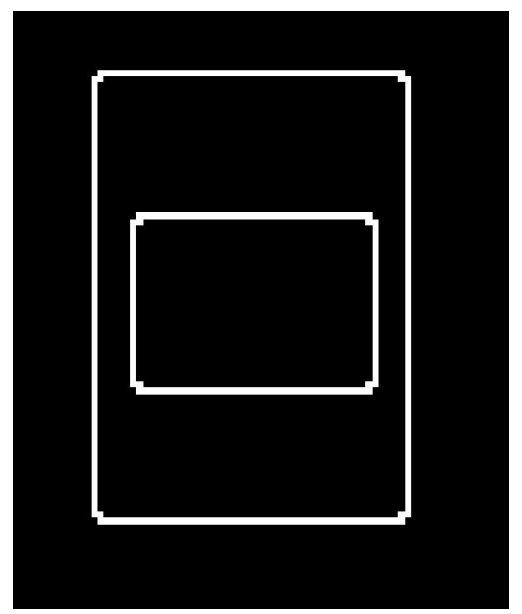

Fig. 7 - Contour detection due to the Canny method

\section{CONCLUSION}

The Canny contour segmentation method was improved using the Haar wavelet transform.

This method has been developed by complementing the original image, using wavelet overlay, threshold filtering and analyzing the extremes.

The developed method allows to detect successfully the contours of the representative image and shows a set of contours in a hierarchical structure.

\section{REFERENCES}

[1] A. C. Davies, S. A. Velastin, "A progress review of intelligent CCTV surveillance systems," in Proceedings of the IEEE Workshop on Intelligent Data Acquisition and Advanced Computing Systems (IDAACS'2005), Sofia, Bulgaria, September 2005, pp. 417-423.

[2] M. Brezovan, C. Badica, "A review on vision surveillance techniques in smart home environments," in Proceedings of the 19th International Conference on Control Systems and Computer Science, 2013, pp. 471-478.

[3] R. Patrick, N. Bourbakis, "Surveillance systems for smart homes: A comparative survey," in Proceedings of the 21st IEEE International Conference on Tools with Artificial Intelligence, 2009, pp. 248-252.

[4] P. V. Kale, S. D. Sharma. "Review of securing home using video surveillance," International Journal of Science and Research (IJSR), Vol. 3, Issue 5, pp. 1150-1154, May 2014.

[5] Oracle White Paper. Dramatically Reduce the Cost and Complexity of Video Surveillance Storage. 2015, [Online]. Available: http://www.oracle.com/us/solutions/oos/tieredstorage-infrastructure/wp-video-surveillancestorage-2288409.pdf

[6] Infortrend Corporation, Safeguarding Data Storage for Video Surveillance and Security, 2006, [Online]. Available: http://infortrend. com/ImageLoader/LoadDoc/167/True/True/Inf ortrend document.

[7] R. C. Gonzalez, R. E. Woods, Digital Image Processing, 3rd Edition, Pearson, 2007, 976 p.

[8] W. K. Pratt. Introduction to Digital Image Processing, 1st Edition, CRC Press, 2013, $756 \mathrm{p}$.

[9] M. V. Poliakova, V. N. Krylov, "The morphological method of a contour segmentation on the basis of regular wavelet transform," Transactions of Odessa Polytechnic University, Vol. 1, Issue 25, pp. 98-103, 2006.

[10] D. Zahorodnia, K. Kovalok, A. Sachenko, V. Krylov, S. Nychyporuk, "Contour 
segmentation method in video surveillance systems," in Proceedings of the International Conference on Modern Problems of Radio Engineering, Telecommunications and Computer Science (TCSET'2014), LvivSlavske, Ukraine, February 25 - March 1, 2014, p. 405.

[11] D. Zahorodnia, Y. Pigovsky, P. Bykovyy, V. Krylov, I. Paliy, I. Dobrotvor, "Structural statistic method identifying facial images by contour characteristic points," in Proceedings of the 8th IEEE International Conference on Intelligent Data Acquisition and Advanced Computing Systems: Technology and Applications (IDAACS'2015), Warsaw, Poland, September 24-26, 2015, pp. 293-297.

[12] D. I. Zahorodnia, P. E. Bykovyy, "Structuralhierarchical principle of contour segmentation," in Current Issues in Modern Technologies: Proceedings of the IV International Scientific and Technical Conference of Young Researchers and Students, Ternopil, Ukraine, November 25-26, 2015, Vol. 2, pp. 20-21.

[13] J. J. Clark, "Authenticating edges produced by zero-crossing algorithms," IEEE Transactions on Pattern Analysis Machine Intelligence, Vol. 12, Issue 8, pp. 830-831, 1989.

[14] P. Marr, M. Doron, "Robust regression for computer vision: a review," International Journal of Computer Vision, Vol. 6, Issue 1, pp. 59-70, 1991.

[15] S. Lakshmi, V. Sankaranarayanan. "A study of edge detection techniques for segmentation computing approaches," International Journal on Computer Applications. Special Issue on Computer Aided Soft Computing Techniques for Imaging and Biomedical Applications, pp. 35-41, 2010.

[16] Y. Ramadevi, "Segmentation and object recognition using edge detection techniques," International Journal of Computer Science and Information Technology, Vol. 2, No. 6, pp. 153-161, 2010.

[17] N. Senthilkumaran, R. Rajesh, "Edge detection techniques for image segmentation. A survey of soft computing approaches," International Journal of Recent Trends in Engineering, Vol. 1, No. 2, pp. 250-254, 2009.

[18] A. Rosenfeld and L. S. Davis, "Image segmentation and image models," Proceedings of IEEE, Vol. 67, No. 5, pp. 764-772, May 1979.

[19] J. E. Canny, "A computational approach to edge detection," IEEE Transactions on Pattern Analysis and Machine Intelligence, Vol. 8, No. 6, pp. 679-698, 1986.

[20] V. Krylov, M. Polyakova, "The method of image contour segmentation based on wavelet

transform and mathematical morphology," in Proceedings of the International Conference on Modern Problems of Radioengineering, Telecommunications and Computer Science (TCSET 2006), Ukraine, 2006, pp. 236-239.

[21] M. Polyakova, V. Krylov, "Image contour segmentation in the space of coefficients of signal semantic wavelet transform," in Proceedings of the International Conference on Modern Problems of Radioengineering, Telecommunications and Computer Science (TCSET 2008), Ukraine, 2008, pp. 280-283.

[22] M. Polyakova, V. Krylov, "Classification of methods of the signal semantic wavelet transform for image contour segmentation," International Journal of Computing, Vol. 7, Issue 1, pp. 51-57, 2008.

[23] R. Porter, N. Canagarajah, "A robust automatic clustering scheme for image segmentation using wavelets", IEEE Transactions on Image Processing, Vol. 5, No. 4, pp. 662-665, 1996.

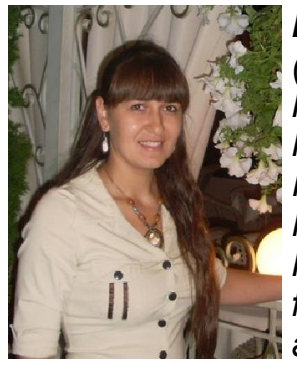

Diana Zahorodnia, Bachelor (2008) and Master (2009), Pedagogic Education, Teacher of Mathematics and Basics of Informatics, V. Hnatiuk Ternopil National Pedagogic University, Ph.D. student (2012), Department for Information Computer Systems and Control, Ternopil National Economic University (2012).

Research interests: images identification, images analysis algorithms for computer recognition systems.

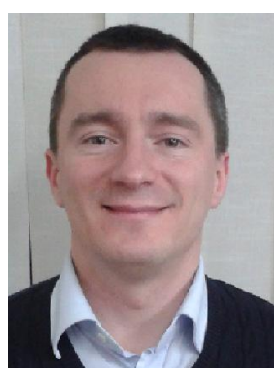

algorithms.

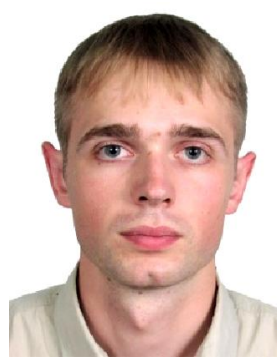

Pavlo Bykovyy, Bachelor (2004), Computer Engineering, Ternopil Academy of National Economy, Specialist (2005), Computer Systems and Networks, IEEE Member (2004), IEEE Student Branch Chairman in Ternopil State Economical University (2005), Ph. D. in Computer Systems and Components (2011). Research interests: security systems, databases, software development. 